\section{Perfis de causas múltiplas de morte relacionadas ao HIV/AIDS nos municípios de São Paulo e} Santos, Brasil, 2001

\author{
Profiles of multiple causes of death related to \\ HIV/AIDS in the cities of São Paulo and Santos, \\ Brazil, 2001
}

\author{
${ }^{1}$ Department of Population \\ Health Sciences, University \\ of Wisconsin Medical School, \\ Madison, U.S.A. \\ 2 Centro de Desenvolvimento \\ e Planejamento Regional, \\ Universidade Federal de \\ Minas Gerais, Belo Horizonte, \\ Brasil. \\ Correspondência \\ C. J. Machado \\ Departamento de \\ Demografia, Centro \\ de Desenvolvimento e \\ Planejamento Regional, \\ Universidade Federal de \\ Minas Gerais. \\ Av. Augusto de Lima 1376 \\ sala 908, Belo Horizonte, $M G$ \\ 30190-003, Brasil. \\ carla@cedeplar.ufmg.br \\ cjmachado@terra.com.br \\ cmachado@jhsph.edu
}

\begin{abstract}
Following the introduction of highly active antiretroviral therapy (HAART), the HIV-related morbidity-mortality profile has changed. Opportunistic infections are not as prevalent as before, and "pre-AIDS" diseases have become more common, related mostly to the side effects of HAART. This study focused on HIVIAIDS-related mortality, based on multiple causes of death among individuals who died of HIV-related causes in the cities of São Paulo and Santos, Brazil, in 2001. Grade of Membership (GoM) analysis was used. Three mortality profiles were detected: (1) causes of death normally observed before the introduction of HAART, marked by opportunistic infections; (2) causes of death with mixed characteristics, both pre-and postHAART; and (3) a residual profile, which did not contemplate HIV disease, but incorporated causes of death associated with the pre and post-HAART periods. It is hoped that the current study will contribute to the understanding of the HIVIAIDS morbidity-mortality scenario and help improve the treatment and care provided by public health services.
\end{abstract}

Highly Active Antiretroviral Therapy; HIV; Acquired Immunodeficiency Syndrome; Multiple Cause of Death
Claudia Cristina de Aguiar Pereira 1

Carla Jorge Machado 2

Roberto do Nascimento Rodrigues 2

\section{Introdução}

Nos últimos anos, a infecção pelo HIV passou a ser considerada como uma doença crônica, com possibilidades de complicações de longo prazo 1. No Brasil, a partir da garantia do acesso à terapia anti-retroviral de alta potência (TARV), houve uma redução da letalidade da doença, o que representou uma queda de $50 \%$ da mortalidade por AIDS no país ${ }^{2}$. Assim, a sobrevida das pessoas com AIDS cresceu 12 vezes, passando de cinco meses, quando do aparecimento da doença e quando não havia tratamento, para quase cinco anos. Dada a evidência de maior sobrevivência, estudos temporais das causas de morte por AIDS, nos períodos pré e pós-terapia antiretroviral têm evidenciado que atenção especial deve ser dada à prevenção de doenças crônicas, que advêm do uso prolongado da terapia anti-retroviral 3. Quando há o uso prolongado da TARV, há um aumento de peso, redistribuição de gordura corporal e obesidade, fatores de risco para uma série de novas comorbidades. Alterações na composição corporal têm sido relatadas, havendo acúmulo de gordura nas regiões centrais do corpo, como tronco, abdômen e região dorso-cervical. Essas alterações estão associadas a doenças cardiovasculares, intolerância à glicose e diabetes mellitus, e diminuição da densidade óssea 4 . Têm sido relatadas ainda mortes relacionadas a problemas no fígado, em pacientes com hepatite tipo B ou C, acometidos pelo HIV. De fa- 
to, infecções oportunistas e neoplasias deixaram de ser as principais causas de morte em alguns casos, sendo substituídas por insuficiência hepática, em geral associadas com infecções pelo vírus da hepatite $\mathrm{C}$ (HCV), e por complicações associadas aos pára-efeitos dos anti-retrovirais, especialmente acidose lática 5,6.

Dessa forma, surge um novo cenário de morbimortalidade e um estudo de causas múltiplas de morte relacionadas ao HIV/AIDS pode promover subsídios para a atuação na prevenção dessas comorbidades, que mudaram ao longo do tempo. Diante desse novo quadro de comorbidades é razoável esperar que um número maior de causas de morte seja observado por indivíduo, quando há utilização da TARV, tanto devido ao aumento de sobrevida com o tratamento, associado ao maior tempo de exposição a doenças, quanto pelo surgimento das novas comorbidades associadas à doença pelo HIV 7. Daí a importância de se utilizar causas múltiplas, pois num contexto de aumento da sobrevida dos portadores do HIV, ou dos indivíduos com AIDS, passa a ser importante descrever e analisar os determinantes patológicos da mortalidade em populações, como complemento ao uso tradicional da causa básica como modelo, que não é capaz de descrever o quadro global do processo mórbido 8,9.

O estudo é feito com base em dados para óbitos de residentes nos Municípios de São Paulo e Santos, Estado de São Paulo, Brasil, 2001. A escolha desses dois municípios se justifica uma vez que, em 1998, o Município de São Paulo detinha o maior número de casos de AIDS no Brasil, ainda que seu peso relativo venha sendo reduzido pouco a pouco devido ao crescimento do número de casos no interior 10. Já o Município de Santos liderou durante anos o ranking de números de casos de AIDS proporcionalmente à população residente. Em meados da década de 90, metade dos casos do município era decorrente do uso de drogas injetáveis. A disseminação da epidemia de HIV/AIDS na população de usuários de drogas já foi bastante relacionada com as rotas escolhidas pelo tráfico para levar as drogas aos mercados da Europa e da América do Norte, sendo o Porto de Santos uma das mais importantes portas de saída das mesmas da América Latina 2.

Fez-se ainda, neste trabalho, um recorte por sexo, pois há um claro diferencial de mortalidade por AIDS entre os sexos 10

Dessa forma, a mortalidade por HIV/AIDS foi focalizada, neste trabalho, a partir das causas múltiplas. Objetivou-se traçar um panorama dos óbitos por HIV/AIDS e das causas de morte que mais freqüentemente aparecem a elas associadas.

\section{Material e métodos}

A fonte de dados utilizada foi o Sistema de Informações sobre Mortalidade (SIM) 11 do Ministério da Saúde, referente aos óbitos de residentes nos municípios de São Paulo e Santos no ano de 2001. No Estado de São Paulo, a cobertura é considerada completa 12 . No período do presente estudo, vigora a 10a revisão da Classificação Internacional de Doenças (CID-10). As informações utilizadas dessas declarações foram sexo, causa básica e causas associadas, que constam nas linhas A, B,C, D, E e ii das declarações de óbito (DO), sendo que, na base de dados, as linhas A, B, C e D podem conter a causa básica, a causa imediata da morte e as causas contributórias para o óbito. As linhas E e ii referem-se ao campo B das DO destinadas à menção de outras condições significativas que contribuíram para o óbito. Para o correto preenchimento da DO é necessário que no mínimo a linha A seja preenchida com a causa básica. Se a causa imediata ou as causas contributórias também são mencionadas, a causa básica é mencionada nas linhas B, C ou D 13. A causa básica recebe um campo específico e é novamente mencionada em uma das linhas $\mathrm{A}, \mathrm{B}, \mathrm{C}$ ou D. Ressalte-se que pode haver mais de uma causa numa mesma linha e que nem todas as linhas são necessariamente preenchidas.

Para selecionar as causas de morte por AIDS e doenças relacionadas, adotou-se o seguinte critério: (1) que na coluna de causas básicas houvesse menção de AIDS (B20-24) ou qualquer uma das doenças da Figura 1, consideradas como altamente indicativas de AIDS ou associadas; (2) ou que os óbitos cujas causas mencionadas nas linhas A até linha ii dos campos do SIM fossem: AIDS (B20-24) ou sarcoma de Kaposi em qualquer localização (C46); ou pneumocistose, que é a pneumonia causada por Pneumocystis carinii (B59), ou imunodeficiência não especificada (D84.9). Observe-se que não foram consideradas as causas associadas da classificação sugerida pelo Ministério da Saúde no seu boletim epidemiológico ${ }^{14}$ quando na causa básica, a qual consta dos campos do SIM, não houvesse quaisquer das causas da mesma classificação, mas somente a doença causada pelo HIV e as doenças altamente indicativas de AIDS. Tal escolha visou a não inclusão de óbitos que não fossem relacionados a AIDS.

Para cada óbito, geralmente, há múltiplas menções de causas. No conjunto desses óbitos puderam ser identificadas 1.715 diferentes causas com códigos alfanuméricos diferenciados que foram agrupadas em 26 variáveis categóricas as quais, por sua vez, foram transformadas em variáveis binárias com o código "1" na presen- 


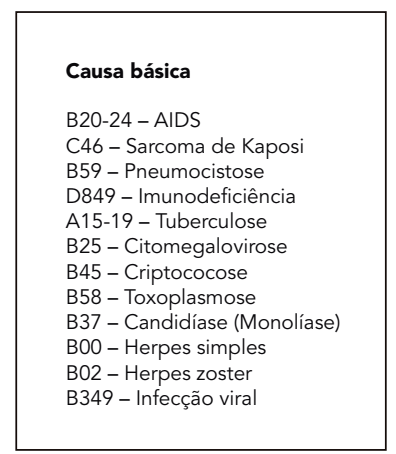

Fonte: Boletim Epidemiológico AIDS 14.

ça das doenças que se enquadraram no agrupamento realizado e " 0 " caso contrário (Tabela 1). Procurou-se respeitar na categorização os grandes agrupamentos, referentes a capítulos da CID-10 e, ao mesmo tempo, tentou-se categorizar as doenças importantes para o estudo do HIV separadamente, como, por exemplo, linfoma não-Hodgkin, sarcoma de Kaposi, hepatites $\mathrm{B}$ e C, e diabetes. Buscou-se, ainda, tratar separadamente doenças que desempenham um papel importante na mortalidade pelo HIV, tanto no período pré-TARV (como a pneumocistose e a caquexia) quanto no período pós-TARV (como a hepatite C). A variável "SY" é uma variável residual, pois nela foram agrupadas doenças que apresentaram freqüências muito pequenas no momento do agrupamento. As demais variáveis categóricas são referentes ao município de ocorrência do óbito (código "1" no caso de São Paulo e " 0 " no caso de Santos) e o sexo da pessoa que faleceu (código " 1 " para sexo masculino e "0" para feminino).

\section{O método Grade of Membership}

O método Grade of Membership (GoM) ou grau de pertinência é uma técnica de classificação multivariada, baseada na teoria dos conjuntos nebulosos 15. A noção de conjuntos nebulosos visa a superar o problema de classificação de um indivíduo de acordo com sua semelhança única e total a um único conjunto com base em critérios e definições que são, em parte, nebulosos ou incompletos. A análise de GoM permite desenvolver perfis ou "tipos ideais" tanto teórica quanto empiricamente. Através dos conjuntos nebulosos, um indivíduo pode ser classificado em diferentes grupos, por meio de escores, para que a heterogeneidade seja expressa de forma parametrizada. Assim, os indivíduos podem fazer parte, simultaneamente, de diversos perfis através de seus escores a cada um dos perfis, que são o grau de pertinência ou a medida da extensão em que o indivíduo pertence a um conjunto nebuloso em particular 16 .

Para que se construa um modelo de GoM, assume-se que o fenômeno estudado é composto de $\mathrm{K}$ conjuntos nebulosos $(\mathrm{k}=1,2, \ldots, \mathrm{K})$ e que a população em estudo possui I indivíduos ( $\mathrm{i}=1$, 2. ..., I). Para cada indivíduo existem J variáveis categóricas mensuradas, onde a j-ésima variável tem $\mathrm{L}_{\mathrm{j}}$ níveis de resposta. Se $\mathrm{x}_{\mathrm{ijl}}=1$, então o i-ésimo indivíduo tem a resposta que corresponde a "1". A variável resposta discreta $\mathrm{x}_{\mathrm{ijl}}$ é predita por dois conjuntos de coeficientes que são gerados: os $\lambda_{\mathrm{kjl}}$ e $\mathrm{g}_{\mathrm{ik}}$. O coeficiente lambda $\left(\lambda_{\mathrm{kjl}}\right)$ é a probabilidade da l-ésima resposta à j-ésima variável estar associada à k-ésima dimensão dos conjuntos nebulosos K, e mostra a extensão em que a variável resposta discreta $\mathrm{x}_{\mathrm{ijl}}$ está associada à k-ésima dimensão. Quando $\lambda_{\mathrm{kjl}}$ é significativamente maior do que a freqüência marginal, isto significa que a estimativa da probabilidade de que uma causa de morte relacionada ao HIV/ AIDS que pertence ao perfil $\mathrm{k}$ se enquadre naquela categoria é, também, significativamente maior que a estimativa da probabilidade marginal e, então, se considera que esta categoria seja característica de uma causa de morte pertencente ao perfil extremo k. Este raciocínio é o mesmo 
Tabela 1

Nome das variáveis causas de morte, códigos e descrições utilizadas para a criação de variáveis binárias.

Nome da

variável
Código da CID-10 A00-A09/A309-

A499/A810-A879/

B00-B09

A15-19

B15 e B189 e B199/

K70-79

B2

B3

B4

B5

B6

B7

B8

C1

C2

D

E1

E2

E3

F

G

I

J1

$\mathrm{J} 2$

$\mathrm{K}$

N

LR

R

SY
B16 e B181

B171 e B182

B20-B24

B25

B37/B39-B49

B58

B59

C00-D48

(exceto C46 e C81-96)

C46 e C81-96

D50-D64/D65-89

E10-14

E40-46

E00-90

F10-F99

G00-G991

1

J12-J18

$\mathrm{J}$

K

$\mathrm{N}$

$\mathrm{O}$ e $\mathrm{P}$ e $\mathrm{Q}$ e R/L-M e outros

R64

S-TN-Y
Descrição

Doenças infecciosas intestinais/Doenças bacterianas/

Infecções virais do sistema nervoso central

Infecções virais com lesões da pele e mucosas

Tuberculose

Hepatite A/Hepatite viral crônica não especificada/

Hepatite viral, não especificada, sem coma

Doenças do fígado

Hepatite B e hepatite crônica viral B sem agente delta

Hepatite C e hepatite viral crônica C

Doença pelo HIV

Citomegalovirose

Candidíase e micose

Toxoplasmose

Pneumocistose

Neoplasias (exceto sarcoma de Kaposi e linfoma não-Hodgkin

e demais neoplasias resultantes do HIV/AIDS)

Sarcoma de Kaposi/Linfoma não-Hodgkin/

Demais neoplasias resultantes do HIV/AIDS

Anemias/Doenças do sangue

Diabetes mellitus

Desnutrição

Doenças endócrinas, nutricionais e metabólicas (exceto diabetes mellitus e desnutrição)

Transtornos mentais decorrentes do uso de álcool/

Decorrentes do uso de drogas

Demais transtornos mentais e comportamentais

Doenças do sistema nervoso

Doenças do aparelho circulatório

Pneumonia

Doenças do aparelho respiratório (exceto pneumonias)

Doenças do aparelho digestivo (exceto fígado)

Doenças do aparelho geniturinário

Gravidez, parto e puerpério/Afecções originadas no período perinatal

Malformações congênitas, deformidades e anomalias

cromossômicas, outros sintomas e outros sinais (exceto caquexia)

Outros (DST/doenças dos olhos/outras doenças por vírus/

doenças por protozoários/doenças infecto-parasitárias)

Doenças da pele/Doenças do sistema osteomuscular

e do tecido conjuntivo

Caquexia

Lesões, envenenamento e algumas outras conseqüências

de causas externas/Causas externas

Fonte: Departamento de Informação e Informática do SUS 11. 
nos caso das variáveis município de residência e sexo. $\mathrm{O} \mathrm{g}_{\text {ik }}$ é o escore para o i-ésimo indivíduo, para cada dimensão $\mathrm{K}$, analiticamente determinada ${ }^{16}$. $O g_{i k}$ descreve o grau em que o i-ésimo indivíduo pertence ao k-ésimo tipo puro. Para cada indivíduo existem tantos valores de $\mathrm{g}_{\mathrm{ik}}$ quantos forem os números de tipos puros identificados pelo modelo, cuja soma é 1. Este coeficiente mostra quão fortemente a característica de um indivíduo está associada ao perfil para qualquer tipo puro gerado. $\mathrm{O} \mathrm{g}_{\mathrm{ik}}$ é o grau de pertinência e descreve a relação do indivíduo com os perfis do grupo descritos no ${ }_{\mathrm{kjl}}$ da k-ésima dimensão. Os valores dos escores $g_{i k}$ variam entre 0 (sem pertinência) e 1 (pertinência total), com soma de 1 para cada indivíduo 16 .

A equação básica do modelo de GoM pode ser descrita utilizando-se o modelo probabilístico: $\operatorname{Pr}\left(\mathrm{x}_{\mathrm{ijl}}=1\right)=\sum g_{i k} \lambda_{k j l}$; onde $\operatorname{Pr}\left(\mathrm{x}_{\mathrm{ijl}}=1\right)$ é a probabilidade de que a i-ésima pessoa tenha a l-ésima resposta como predito pelo produto interno dos $\mathrm{K}$ pares de gik e $\lambda \mathrm{kjl}$ estimados.

Os parâmetros da função de GoM, gik $\lambda_{\mathrm{kj}}$, são estimados pela maximização da função de verossimilhança multinominal: $\mathrm{L}=$ $\prod_{i} \prod_{i} \prod_{l}\left(\sum_{k=1}^{k} g_{i k} \lambda_{k j l}\right)^{x_{i j}}$.

Ós parâmetros se encontram sob as seguintes restrições: $0 \leq g_{i k} \leq 1 \mathrm{e} \sum_{k} g_{i k}=1$. O procedimento de GoM determina simultaneamente a identidade do subgrupo nebuloso na população e os perfis de atributos que definem os subgrupos 16 .

Neste trabalho, considerou-se que se um óbito tivesse pertinência a um perfil extremo que fosse superior a 0,50 , mas inferior a 1,0, este seria considerado como tendo características predominantes do perfil em questão (mas não seria um tipo puro). Dessa forma, buscou-se obter qual o perfil seria considerado de maior predominância na população estudada. A análise desses óbitos, e não apenas dos óbitos considerados tipos puros, foi realizada neste estudo. Observe-se, no entanto, que se não se estiver no caso específico de dois perfis, é possível que tenhamos óbitos considerados amorfos, ou seja, que não se enquadram nesta classificação, e são considerados eqüidistantes a todos os perfis. Seria o caso, por exemplo, de um modelo de três perfis, onde um óbito teria $\mathrm{g}_{\mathrm{i} 1}=0,3 ; \mathrm{g}_{\mathrm{i} 2}=0,34 ; \mathrm{g}_{\mathrm{i} 3}=0,36$; neste caso, os amorfos configuram-se em uma categoria à parte.

Quanto ao número de perfis do modelo, não existe um procedimento padrão para determiná-los 17. Existem critérios estatísticos na determinação dos perfis, mas, neste estudo, os critérios foram definidos com base na avaliação da "significância substantiva" dos perfis extremos. A cada novo perfil criado procurou-se verificar se as mudanças observadas podiam ser justificadas do ponto de vista teórico 18 , uma vez que o mé- todo de GoM possibilita a obtenção do número de perfis desejado. Cabe observar que no caso do método de componentes principais é amplamente utilizada e aceita a seleção do número de componentes com base no critério de "perfis interpretáveis" (que é o mesmo que "significância substantiva”), o qual se constitui em um critério empírico, mas não desprovido de sentido. O método de GoM é um método similar ao de componentes principais e que visa a reduzir a dimensionalidade dos dados originais, logo julgouse adequada a utilização de um critério também similar 19,20.

Foi utilizado o programa GoM3 executável em ambiente DOS. O programa encontra-se disponível pela Internet (http://www.stat.unipg.it/ stat/statlib/DOS/general/). Utilizou-se a opção "gradiente" (declive máximo), que utiliza processos de otimização para a resolução de sistemas de equações, tomando como base o fato de que quando as funções são regulares, um ponto de mínimo relativo é um ponto crítico que anula a derivada ou o gradiente 21 .

\section{Resultados}

O método multivariado GoM possibilitou a definição de perfis multidimensionais de causas e permitiu verificar as pertinências a cada um dos perfis. De forma resumida, observa-se que foram identificados três perfis, os quais serão comentados mais detalhadamente: perfil 1, o qual aglomerou as causas de morte notadamente identificadas na literatura como típicas do período pré-TARV; perfil 2, característico de causas dos períodos pré e pós-TARV foi determinado por um conjunto maior de doenças; por último, o perfil 3 pôde ser identificado como residual, no qual não estava presente a doença pelo HIV, e poderia captar casos em que a doença pelo HIV não foi mencionada explicitamente, mas somente outras doenças altamente indicativas de HIV.

Das 26 variáveis referentes às causas de morte, quatro delas tiveram freqüência nula. Assim, foram obtidas 22 variáveis binárias de causas de morte de freqüência não nula correspondentes a 6.091 causas para os Municípios de São Paulo e Santos, distribuídas por 1.963 óbitos (1.820 referentes a São Paulo e 143 a Santos), configurando uma média de 3,1 diagnósticos por óbito.

Analisou-se a significância substantiva de modelos com dois, três e quatro perfis. Os resultados obtidos das aplicações do modelo com três perfis foram considerados logicamente mais "interpretáveis” relativamente àqueles que consideraram dois ou quatro perfis do ponto de vista do esperado com base na literatura sobre o assunto. 
Na Tabela 2 encontram-se as freqüências absoluta e relativa das 24 variáveis utilizadas no estudo e as estimativas de $\lambda_{\text {kil }}$ para os três perfis extremos. As características de cada um destes perfis extremos foram obtidas comparando $\lambda_{\mathrm{kj}}$ com a freqüência marginal das respostas na mesma categoria.

É possível verificar como a ocorrência dessas 22 causas de morte e as variáveis de município de ocorrência do óbito e sexo aparecem aglomeradas em perfis. Cada perfil representa diferentes associações entre as causas de morte. Considerou-se que uma categoria l, de uma variável j, seria característica de um perfil $\mathrm{k}$, se $\lambda_{\mathrm{kjl}}$ dividido pela freqüência marginal fosse igual ou maior que 1,2; com exceção da variável município para a qual se aceitou o valor de 1,08. Para a variável município este critério se justifica uma vez que a freqüência de ocorrência de óbitos em São Paulo é muito elevada (92,7\%). Estes valores escolhidos parecem captar adequadamente as características dominantes dos perfis extremos.

O perfil 1 é o de grupos de causas de morte para o qual se observa o aumento da probabilidade de ocorrência das seguintes causas de morte, em relação à população: tuberculose; doença pelo HIV; candidíase e micose; toxoplasmose; pneumonia e doenças do aparelho respiratório (excluindo pneumonias), e ser residente no $\mathrm{Mu}$ nicípio de São Paulo. Logo, se um óbito é um tipo puro do perfil 1 , a probabilidade de que tal óbito apresente quaisquer das causas anteriormente mencionadas é maior do que a de um óbito ocorrido na população total estudada, selecionado ao acaso. Exemplificando, pode-se afirmar que o fato de a tuberculose estar presente no perfil 1 indica que, das pessoas com pertinência total a este perfil $\left(g_{i 1}=1\right)$, espera-se que $44,5 \%$ delas apresentem tuberculose nos campos do SIM, ao passo que a freqüência marginal relativa da tuberculose no total da população é de $29 \%$. De outro modo, pode-se dizer que um indivíduo com escore $\mathrm{g}_{\mathrm{i} 1}=1$ possui uma probabilidade de ser acometido pela tuberculose de 0,445 , enquanto um indivíduo tomado ao acaso na população apresenta probabilidade inferior, de 0,290.

Quanto à denominação desse perfil, por ser constituído pelas causas mais comumente associadas à mortalidade por HIV/AIDS no período pré-TARV, principalmente devido à presença de doenças oportunistas observadas na história natural da doença, como tuberculose, candidíase e micose, e toxoplasmose, chamou-se esse perfil de "perfil típico da mortalidade por HIV, pré-TARV".

O perfil 2 é o perfil de grupos de causas de morte no qual se observa o aumento da probabilidade de ocorrência das seguintes causas em relação à população: tuberculose; hepatite A e virais e doenças do fígado; doença pelo HIV; citomegalovirose; candidíase e micose; toxoplasmose; neoplasias (não diretamente relacionadas ao HIV, ou seja, exceto sarcoma de Kaposi e linfoma não-Hodgkin); neoplasias: sarcoma de Kaposi e linfoma não-Hodgkin; anemias e doenças do sangue; diabetes; desnutrição; demais doenças endócrinas, nutricionais e metabólicas; doenças do sistema nervoso; doenças do aparelho digestivo (exceto do fígado); doenças do aparelho geniturinário; doenças relacionadas à gravidez, parto e puerpério; afecções originadas no período perinatal; malformações congênitas, deformidades e anomalias cromossômicas e outros sinais e sintomas (exceto caquexia); causas externas; ser do sexo masculino e ser residente de Santos. Assim, se um óbito é tipo puro desse perfil, a probabilidade de que o mesmo apresente quaisquer das doenças listadas é maior do que a de um óbito selecionado ao acaso nessa população. Esse perfil foi denominado "perfil típico de associações de doenças pré e pós-TARV”.

O perfil 3 é o perfil cujo agrupamento de causas é o seguinte: doenças infecciosas intestinais/ doenças bacterianas/infecções virais do sistema nervoso central/infecções virais com lesões da pele e mucosas; transtornos mentais decorrentes do uso de álcool/decorrentes do uso de drogas/ demais transtornos mentais e comportamentais; doenças do aparelho circulatório; doenças do aparelho respiratório (exceto pneumonias), e ser residente no Município de São Paulo. Esse perfil foi denominado de "perfil de doenças não relatadas como diretamente relacionadas ao HIV/AIDS”.

Quanto à predominância das características dos perfis na população, o perfil extremo 1 foi delineado com base em $12,4 \%$ da população (243 óbitos), referentes aos óbitos que apresentam escores $g_{i 1}$ iguais a um; já os perfis extremos 2 e 3 representam $6,4 \%$ (126 óbitos) e 9,6\% (188 óbitos) da população, respectivamente, com escores $g_{i 2}$ e $g_{i 3}$ iguais a $u m$. Assim, verifica-se que a predominância dos perfis extremos na população é de apenas 28,4\%, (557 óbitos), ou seja, 71,6\% (1.406 óbitos) não são tipos puros de qualquer perfil. Destes 1.406 óbitos, 451 tinham características predominantes do perfil 1 ( $\mathrm{g}_{\mathrm{i} 1}$, maior que 0,5 e inferior a 1,0), representando $22,9 \%$ do total; encontrou-se também que 460 óbitos tinham características predominantes do perfil 2 (23,6\% do total); e, finalmente, 372 óbitos tinham características predominantes do perfil 3 (19\% do total). Note-se que foram encontrados 123 óbitos eqüidistantes a todos os perfis extremos, ou seja, que não possuem características que os aproximem de qualquer perfil específico (6,3\% dos óbitos). 
Estimativas de $\lambda_{\mathrm{kjl}}$ segundo perfis extremos e categorias das variáveis e freqüências, referentes às causas múltiplas de morte.

Municípios de São Paulo e Santos, São Paulo, Brasil, em 2001.

\begin{tabular}{|c|c|c|c|c|c|c|c|}
\hline \multirow[t]{2}{*}{ Código } & \multirow[t]{2}{*}{ Variáveis } & \multirow[t]{2}{*}{ Categoria } & \multicolumn{2}{|c|}{ Freqüência marginal } & \multicolumn{3}{|c|}{ Perfis extremos } \\
\hline & & & Absoluta & Relativa & $1\left(\lambda_{1 \mathrm{j}}\right)$ & $2\left(\lambda_{2 \mathrm{j}}\right)$ & $3\left(\lambda_{3 \mathrm{j} j}\right)$ \\
\hline \multirow[t]{2}{*}{ MUN } & Santos & 0 & 143 & 0,073 & 0,000 & 0,226 & 0,000 \\
\hline & São Paulo & 1 & 1.820 & 0,927 & 1,000 & 0,774 & 1,000 \\
\hline \multirow[t]{3}{*}{ A1 } & Doenças infecciosas/Intestinais/Bacterianas/ & 0 & 1.301 & 0,663 & 1,000 & 1,000 & 0,0000 \\
\hline & Infecções virais do sistema nervoso central/ & 1 & 662 & 0,337 & 0,000 & 0,000 & 1,000 \\
\hline & Com lesões da pele e mucosas & & & & & & \\
\hline \multirow[t]{2}{*}{ A2 } & Tuberculose & 0 & 1.394 & 0,710 & 0,555 & 0,601 & 1,000 \\
\hline & & 1 & 569 & 0,290 & 0,445 & 0,399 & 0,000 \\
\hline \multirow[t]{2}{*}{ B1 } & Hepatite A/Hepatite viral crônica não especificada, & 0 & 1.861 & 0,938 & 1,000 & 0,806 & 1,000 \\
\hline & hepatite viral não especificada sem coma/Doenças do fígado & 1 & 122 & 0,062 & 0,000 & 0,194 & 0,000 \\
\hline \multirow[t]{2}{*}{ B4 } & Doença pelo HIV & 0 & 572 & 0,291 & 0,000 & 0,000 & 1,000 \\
\hline & & 1 & 1.391 & 0,709 & 1,000 & 1,000 & 0,000 \\
\hline \multirow[t]{2}{*}{ B5 } & Citomegalovirose & 0 & 1.937 & 0,987 & 0,992 & 0,969 & 1,000 \\
\hline & & 1 & 26 & 0,013 & 0,008 & 0,031 & 0,000 \\
\hline \multirow[t]{2}{*}{ B6 } & Candidíase e micose & 0 & 1.831 & 0,933 & 0,817 & 1,000 & 1,000 \\
\hline & & 1 & 132 & 0,067 & 0,183 & 0,000 & 0,000 \\
\hline \multirow[t]{2}{*}{ B7 } & Toxoplasmose & 0 & 1.781 & 0,907 & 0,749 & 1,000 & 1,000 \\
\hline & & 1 & 182 & 0,093 & 0,251 & 0,000 & 0,000 \\
\hline \multirow[t]{2}{*}{ C1 } & Neoplasias (exceto sarcoma de Kaposi e linfoma & 0 & 1.822 & 0,928 & 1,000 & 0,775 & 1,000 \\
\hline & não-Hodgkin e demais neoplasias resultantes do HIV/AIDS) & 1 & 141 & 0,072 & 0,000 & 0,225 & 0,000 \\
\hline \multirow[t]{2}{*}{$\mathrm{C} 2$} & Sarcoma de Kaposi/Linfoma não-Hodgkin/ & 0 & 1.926 & 0,981 & 1,000 & 0,775 & 1,000 \\
\hline & Demais neoplasias resultantes do HIV/AIDS & 1 & 37 & 0,019 & 0,000 & 0,225 & 0,000 \\
\hline \multirow[t]{2}{*}{ D } & Anemias/Doenças do sangue & 0 & 1.921 & 0,979 & 1,000 & 0,933 & 1,000 \\
\hline & & 1 & 42 & 0,021 & 0,000 & 0,067 & 0,000 \\
\hline \multirow[t]{2}{*}{ E1 } & Diabetes mellitus & 0 & 1.820 & 0,927 & 1,000 & 0,774 & 1,000 \\
\hline & & 1 & 143 & 0,073 & 0,000 & 0,226 & 0,000 \\
\hline \multirow[t]{2}{*}{ E2 } & Desnutrição & 0 & 1.922 & 0,979 & 1,000 & 0,935 & 1,000 \\
\hline & & 1 & 41 & 0,021 & 0,000 & 0,065 & 0,000 \\
\hline \multirow[t]{2}{*}{ E3 } & Doenças endócrinas, nutricionais e metabólicas & 0 & 1.906 & 0,971 & 1,000 & 0,909 & 1,000 \\
\hline & & 1 & 57 & 0,029 & 0,000 & 0,0907 & 0,000 \\
\hline \multirow[t]{2}{*}{$\mathrm{F}$} & Transtornos mentais decorrentes do uso de álcool/ & 0 & 16.889 & 0,962 & 1,000 & 1,000 & 0,880 \\
\hline & Drogas/Demais transtornos mentais e comportamentais & 1 & 74 & 0,038 & 0,000 & 0,000 & 0,120 \\
\hline G & Doenças do sistema nervoso & 0 & 1.830 & 0,932 & 1,000 & 0,790 & 1,000 \\
\hline & & 1 & 133 & 0,068 & 0,000 & 0,210 & 0,000 \\
\hline 1 & Doenças do aparelho circulatório & 0 & 1.773 & 0,903 & 1,000 & 1,000 & 0,699 \\
\hline & & 1 & 190 & 0,097 & 0,000 & 0,000 & 0,301 \\
\hline $\mathrm{J} 1$ & Pneumonia & 0 & 1.355 & 0,690 & 0,000 & 1,000 & 1,000 \\
\hline & & 1 & 608 & 0,310 & 0,000 & 0,000 & 0,000 \\
\hline $\mathrm{J} 2$ & Doenças do aparelho respiratório (exceto pneumonias) & 0 & 1.153 & 0,587 & 0,445 & 1,000 & 0,322 \\
\hline & & 1 & 810 & 0,413 & 0,555 & 0,000 & 0,678 \\
\hline K & Doenças do aparelho digestivo (exceto doenças do fígado) & 0 & 1.853 & 0,944 & 1,000 & 0,825 & 1,000 \\
\hline & & 1 & 110 & 0,056 & 0,000 & 0,175 & 0,000 \\
\hline $\mathrm{N}$ & Doenças do aparelho geniturinário & 0 & 1.827 & 0,931 & 0,966 & 0,827 & 1,000 \\
\hline & & 1 & 136 & 0,069 & 0,034 & 0,173 & 0,000 \\
\hline LR & Gravidez, parto e puerpério/Outros sintomas e outros sinais & 0 & 1.507 & 0,768 & 1,000 & 0,322 & 1,000 \\
\hline & & 1 & 456 & 0,232 & 0,000 & 0,678 & 0,000 \\
\hline SY & Lesões, envenenamento e algumas outras conseqüências & 0 & 1.934 & 0,985 & 1,000 & 0,954 & 1,000 \\
\hline & de causas externas/Causa externa & 1 & 29 & 0,015 & 0,000 & 0,462 & 0,000 \\
\hline SEX & Sexo masculino & 0 & 612 & 0,321 & 0,566 & 0,000 & 0,285 \\
\hline & & 1 & 1.351 & 0,688 & 0,434 & 1,000 & 0,715 \\
\hline
\end{tabular}

Fonte: Departamento de Informação e Informática do SUS 11. 


\section{Discussão}

As análises referentes aos perfis encontrados mostram-se bastante próximas dos resultados encontrados na literatura. As evidências são de que novas doenças, principalmente as pré-AIDS, vêm aparecendo com maior freqüência, embora não tenham sido erradicadas as doenças oportunistas da história natural do HIV, conforme o resultado do perfil 2, no qual foram encontradas doenças como diabetes associadas a doenças oportunistas. Observou-se ainda a persistência das doenças pré-TARV, identificadas na história natural do HIV. Este trabalho demonstra, assim como outros, que há pouca evidência de que as doenças pré-TARV não continuem existindo no período pós-TARV 22.

No caso do perfil 3, o aparecimento sistemático das causas que aparecem conjuntamente nesses perfis merece futuras investigações, uma vez que os cinco grupos de causas deste perfil (doenças infecciosas, bacterianas e infecções; transtornos mentais e comportamentais; doenças do sistema nervoso; doenças do aparelho circulatório e doenças do aparelho respiratório) no contexto da doença pelo HIV são indícios de óbitos em pacientes em estágio avançado de AIDS 23,24.

$O$ perfil extremo de maior peso no total da população é o perfil 1, constituído pelas doenças oportunistas, onde se enquadram $35,3 \%$ dos óbitos como tipos puros ou predominantes. Note-se que um óbito tipo puro desse perfil necessariamente ocorreu no Município de São Paulo (uma vez que, se um óbito é tipo puro deste perfil, ele tem $100 \%$ de probabilidade de ser de um residente do Município de São Paulo). Esse resultado indica que, nesse município, continua-se observando um agrupamento de doenças típicas do período pré-TARV. Esse ponto merece futuras investigações com o objetivo de identificar se esses óbitos são decorrentes de pessoas que apresentaram falha terapêutica ou se houve problemas de adesão ao tratamento, ou até mesmo se a TARV não chegou a ser utilizada por questões de acesso, num contexto em que se espera que no Município de São Paulo a TARV seja universalmente utilizada, quando necessário. Por outro lado, mesmo diante da utilização bem sucedida dos medicamentos, após determinado período de tempo, a medicação pode se tornar incapaz de sustentar uma carga viral baixa e uma contagem de células T-CD4+ acima de 200células $/ \mathrm{mm}^{3}$, ou seja, o nível considerável "não AIDS” 25. Esses são questionamentos que os pesquisadores do HIV vêm levantando.

No que diz respeito ao perfil 2, houve uma maior aglomeração de causas tanto observáveis no período pré quanto pós-TARV. Tal resultado pode estar relacionado ao fato de alguns desses óbitos se referirem a pacientes que iniciaram a terapia anti-retroviral de alta potência já com uma contagem de células T-CD4+ baixa e que, portanto, faleceram não muito tempo após o início de sua utilização. Por outro lado, podem não ter tido tempo suficiente para adquirirem doenças típicas dos efeitos adversos da utilização dos esquemas triplos da TARV, como diabetes mellitus e lipodistrofia. Mais uma vez, a possibilidade, que precisa ser investigada de forma mais aprofundada, é a questão do acesso à terapia e adesão dos pacientes a mesma. De fato, se há falhas nesses dois processos (acesso e adesão), a progressão do paciente da infecção até a AIDS pode ocorrer mais rapidamente, com manifestação de infecções oportunistas observadas na história natural do HIV, e que certamente podem levar ao óbito dos indivíduos em período curto.

No perfil 2 foram verificadas comorbidades que podem estar associadas ao uso de drogas injetáveis, no Município de Santos, que é um dos municípios com maior taxa de soroprevalência de HIV entre usuários de drogas injetáveis no mundo. Esta associação entre uso de drogas injetáveis e HIV está também relacionada à rota dos caminhoneiros para o Porto de Santos e à prostituição 26. Cabe ainda observar o fato de o sexo masculino ser uma característica desse perfil, o que parece indicar uma provável conseqüência da maior vulnerabilidade dos usuários de drogas injetáveis. Além disso, alguns estudos também indicam que há diferencial por sexo na freqüência e severidade dos efeitos adversos da TARV. Dentre os efeitos adversos relatados, os mais freqüentes são: acidose lática, feridas, elevação do número de enzimas do fígado, dislipidemia e resistência à insulina 27,28. Todas essas doenças se enquadram nos agrupamentos de doenças encontrados no perfil 2.

Os resultados relativos aos óbitos com características predominantes dos perfis 1, 2 ou 3 merecem algumas considerações. Em primeiro lugar, é de se esperar que, devido ao grande número de possíveis comorbidades existentes entre aqueles que morrem em decorrência da infecção pelo HIV, que se depare com este tipo de situação, na qual um determinado óbito não pertence exclusivamente a um dos perfis, tendo também grau de pertinência menor a outros perfis. Tal afirmação está baseada na literatura, acerca das diversas causas que podem acometer esses indivíduos, e que podem aparecer em conjunto. Logo, o fato de nem todos os óbitos serem tipos puros de um ou outro perfil não é um evento inesperado. Em segundo lugar, é importante ressaltar que, para 93,7\% dos óbitos (1.839 dos 1.963 óbitos) foi 
encontrada predominância a um ou outro perfil, ou seja, existe um baixo percentual de amorfos nessa população, o que indica que os perfis adequadamente conseguiram captar as principais características daqueles que faleceram em virtude do HIV/AIDS, conforme critério deste estudo. A idéia é que se houvesse um número elevado de amorfos, não se teria conseguido sintetizar, para muitos óbitos, quais as suas principais características definidoras.

Uma limitação deste trabalho diz respeito ao critério de seleção das causas. Utilizou-se uma recomendação do boletim epidemiológico do Ministério da Saúde 14, havendo outras possibilidades que não alterariam significativamente o produto final. Outra questão está relacionada aos agrupamentos de doenças, que, por um lado, facilitaram o trabalho, mas, por outro, impossibilitaram visualizar algumas doenças específicas isoladamente. Tentou-se amenizar este problema ao se tratar separadamente doenças como a citomegalovirose e as neoplasias relacionadas ao HIV. A tuberculose pulmonar e não pulmonar foram tratadas conjuntamente, o que pode ter levado à seleção de pessoas que não são portadoras do HIV, por ser uma doença de alta prevalência no Brasil. Além disso, a literatura mostra que a tuberculose pulmonar é o tipo de tuberculose mais freqüente em pessoas, sejam HIV positivas ou não 29 . No que diz respeito às neoplasias, futuros estudos poderiam desmembrar o agrupamento realizado, procurando observar alguns tipos específicos de câncer, como o cervical e o anal, para que melhor se apreenda a relação entre a mortalidade pelo HIV, as neoplasias e o uso da TARV.

Um corte por idade teria sido útil no estudo de algumas doenças que estão mais relacionadas à idade do infectado pelo HIV, como por exemplo algumas doenças do sistema nervoso, de maior prevalência em pacientes idosos. Não se realizou um recorte por grupos etários neste trabalho, mesmo diante do conhecimento de que a história natural do HIV é diferente para crianças e adultos, pois o número de óbitos de menores de 13 anos foi muito pequeno.

Uma informação, certamente útil no caso deste estudo, seria se os pacientes foram diagnosticados há muito tempo ou se foram submetidos a vários esquemas de tratamento. Isso possibilitaria validar os perfis, verificando se de fato os conjuntos de causas encontrados correspondem, de fato, ao esperado. Por exemplo, uma probabilidade de pertinência elevada ao perfil 1, de doenças pré-TARV seria condizente com uma probabilidade aumentada de diagnóstico antigo e tratamento com AZT, para os quais a TARV é menos eficaz 30 .
Quanto ao uso do GoM como método de análise, devem ser feitas algumas considerações. Em primeiro lugar, o método adequou-se ao objetivo uma vez que este procedimento analítico ajudou na sintetização de um número bastante grande de variáveis - as causas de óbito - em um número considerado razoável de grupos distintos, consistentes com o esperado com base na literatura sobre o assunto, conforme já discutido, o que valida os resultados obtidos neste trabalho. Além disso, consegue-se lidar com a questão da heterogeneidade entre os indivíduos. Por exemplo, no caso do presente estudo, ainda que dois indivíduos possam ser acometidos por pneumonia com probabilidade de $100 \%$, a probabilidade de que um tenha tido também tuberculose pode ser bastante superior a do outro, o que indica que a variação de combinações de morbidades entre indivíduos tende a não ser um caso atípico. A constatação dessas associações não seria possível por meio de um estudo meramente descritivo. De fato, para análise de causas múltiplas, os estudos de associações de causas são ainda de uso restrito e estudos baseados na independência das variáveis não se aplicam às análises de associações de causas, dado serem interdependentes. Dessa forma, o método GoM revelou-se um procedimento metodológico pertinente ao objetivo. Em segundo lugar, vale comentar que o programa de computador utilizado é um software especializado, destina-se a uma atividade com conteúdo preciso, bastando conhecer o modelo a fim de que se possa interpretar os resultados. É de fácil utilização e aprendizado e de fácil memorização de informações importantes para o uso. A documentação sobre o uso do programa e sobre a instalação é de fácil compreensão. Por último, um aspecto que merece ser ressaltado, muito embora não se tenha constituído em um problema no caso do presente estudo, refere-se às limitações do modelo. Pode-se pensar que o modelo parte da premissa básica de que existe algo comum entre os indivíduos, e é isto que permite a definição de perfis. Assim, quando se está diante de um pequeno número de indivíduos, muito heterogêneos, seria esperada maior dificuldade em se encontrar um determinado conjunto de comorbidades que se reiterasse com freqüência. Logo, quanto menor o tamanho do universo estudado, maior seria o estado de entropia, e seria muito difícil de se encontrar um número de perfis que fosse inferior ao número de indivíduos 16 . Nesse caso, o método teria pouca ou nenhuma utilidade, além de uma mera análise descritiva dos indivíduos. 


\section{Conclusão}

Este trabalho teve como motivação a tentativa de abordar as causas múltiplas de morte relacionadas ao HIV/AIDS. Considerou-se adequada a aplicação do método de GoM, pois o mesmo possibilitou a definição de três perfis de causas, ao mesmo tempo em que permitiu analisar as diferentes intensidades de pertinência a cada um dos perfis criados.

Do ponto de vista de políticas públicas é importante perceber o perfil da mortalidade pelo HIV/AIDS para que se possa enfrentar os desafios da doença como uma doença crônica, pois, apesar do grande decréscimo nas taxas de mortalidade relacionadas ao HIV/AIDS, a doença continua sendo uma das principais causas de morte entre os jovens adultos nos grupos etários entre $25 \mathrm{e}$ 44 anos. Assim, um dos aspectos importantes é a prevalência da doença na população, que pode estar crescendo. Num curto espaço de tempo, a AIDS passou de uma doença fatalmente rápida e uniforme para uma doença controlável diante do evento da TARV. Mesmo no Brasil, onde o trata-

\section{Resumo}

Após a introdução da terapia anti-retroviral de alta potência (TARV), o perfil da morbimortalidade relacionada ao HIV alterou-se, passando de óbitos causados por doenças oportunistas para quadros mórbidocrônicos de doenças "pré-AIDS", ou não definidoras de AIDS, causadas pelos efeitos adversos da terapia. Investigou-se a mortalidade relacionada ao HIVIAIDS através das causas múltiplas de morte, utilizando-se as declarações de óbito de residentes nos municípios de São Paulo e Santos, Brasil, que faleceram de causas relacionadas à doença pelo HIV em 2001. Utilizou-se o método Grade of Membership (GoM), que possibilitou criar perfis de causas de morte. Foram encontrados três perfis de mortalidade: o primeiro, relacionado às causas de morte identificadas no período pré-TARV, com predominância de doenças oportunistas; no segundo, houve uma mistura de características do período prée pós-TARV; o terceiro perfil, residual, não contemplou a doença pelo HIV, mas incorporou grupos de causas de morte associadas aos períodos pré e pós-TARV. Esperase que este estudo contribua para a elaboração de políticas direcionadas à adequação dos serviços de saúde ao novo cenário de morbimortalidade relacionada ao HIV.

Terapia Anti-Retroviral de Alta Atividade; HIV; Síndrome de Imunodeficiência Adquirida; Causas Múltiplas de Morte mento é universal, existem incertezas envolvendo a terapia, adesão, prognóstico e iatrogênesis. Em vez de ser uma doença consideravelmente previsível, estereotipada e com um curso fatal, a AIDS passou a ser uma doença prolongada com exacerbações e remissões, com uma carga de doença cumulativa crescente, com efeitos tóxicos significativos advindos da TARV, com crescentes comorbidades médicas e psiquiátricas 7 .

A utilização da TARV altera o curso da doença pelo HIV e posterga a mortalidade. Entretanto, esses resultados positivos implicam um novo custo e novas demandas, principalmente em termos de morbidade e atenção específica à saúde dos portadores do HIV. Espera-se que os resultados deste estudo contribuam para a elaboração de políticas públicas direcionadas à adequação dos serviços de saúde ao novo cenário de morbimortalidade relacionada ao HIV, no qual se continua observando causas de morte conhecidas ao longo de mais de duas décadas da epidemia do HIV/AIDS juntamente a novas doenças, que em sua maior parte são doenças sérias e crônicas, advindas da utilização da TARV.

\section{Colaboradores}

C. C. A. Pereira, C. J. Machado e R. N. Rodrigues participaram da revisão de literatura, elaboração da metodologia, análise dos resultados e redação do artigo final. 


\section{Referências}

1. Klein SK, Slim EJ, De Kruif MD, Keller TT, ten Cate $\mathrm{H}$, van Gorp EC, et al. Is chronic HIV infection associated with venous thrombotic disease? A systematic review. Neth J Med 2005; 63:129-36.

2. Ministério da Saúde. História da AIDS. http://www. aids.gov.br/data/Pages/LUMIS232EC481PTBRIE. htm (acessado em 03/Ago/2004).

3. Louie JK, Hsu LC, Osmond DH, Katz MH, Schwarcz SK. Trends in causes of death among persons with acquired immunodeficiency syndrome in the era of highly active antiretroviral therapy, San Francisco, 1994-1998. J Infect Dis 2002; 186:1023-7.

4. Jaime PC, Florindo AA, Latorre MRDO, Brasil BG, Santos ECM, Segurado AAC. Prevalência de sobrepeso e obesidade abdominal em indivíduos portadores de HIV/AIDS, em uso de terapia anti-retroviral de alta potência. Rev Bras Epidemiol 2004; 7:65-72.

5. Rachid M, Schechter M. Manual de HIV/AIDS. Rio de Janeiro: Editora Revinter; 2000.

6. Mocroft A, Brettle R, Kirk O, Blaxhult A, Parkin JM, Antunes F. Changes in the cause of death among HIV positive subjects across Europe: results from the EuroSIDA study. AIDS 2002; 16:1663-71.

7. Selwyn PA, Forstein M. Overcoming the false dichotomy of curative vs palliative care for late-stage HIV/AIDS: "Let me live the way I want to live, until I can't". JAMA 2003; 290:806-14.

8. Santo AH, Pinheiro CE. Tabulador de causas múltiplas de morte. Rev Bras Epidemiol 1999; 2:90-7.

9. Machado CJ. Mortalidade infantil no Estado de São Paulo, 1999: uma análise das causas múltiplas de morte a partir de componentes principais. Rev Bras Epidemiol 2004; 7:11-21.

10. Waldwogel BC, Morais LCC. Mudanças no padrão da mortalidade por aids no Estado de São Paulo. http://www.abep.nepo.unicamp.br/docs/anais/ pdf/2002/GT_SAU_ST35_Waldvogel_texto.pdf (acessado em 10/Fev/2004).

11. Departamento de Informação e Informática do SUS. Sistema de informações de nascidos vivos de 1998. http://tabnet.datasus.gov.br/cgi/sinasc/ dados/ (acessado em 22/Jan/2003).

12. Camargo ABM. Mortalidade por causas externas no Estado de São Paulo e suas regiões [Tese de Doutorado]. São Paulo: Faculdade de Saúde Pública, Universidade de São Paulo; 2002.

13. World Health Organization. Official updates to ICD-10. http://www.who.int/entity/classifications /icd/ICD10Updates_2001.pdf (acessado em 10/ Mai/2005).

14. Ministério da Saúde. Boletim Epidemiológico AIDS 2002; Ano XVI, no. 1.

15. Zadeh A. Fuzzy sets. Information and Control 1965; 8:338-53.
16. Manton KG, Woodbury MA, Tolley HD. Statistical applications using fuzzy sets. New York: John Wiley \& Sons; 1994.

17. Berkman L, Singer B, Manton K. Black/white differences in health status among the elderly. Demography 1989; 26:661-78.

18. Sawyer DO, Leite IC, Alexandrino R. Perfis de utilização dos serviços de saúde. Ciênc Saúde Coletiva 2002; 7:757-76.

19. Abbad G, Meneses PPM. Lócus de controle: validação de uma escala em situação de treinamento. Estud Psicol (Natal) 2004; 9:441-50.

20. Fleck MPA, Bourdel MC. Método de simulação e a escolha de fatores na análise dos componentes principais. Rev Saúde Pública 1998; 32:267-72.

21. Cerqueira CA. Tipologia e características dos estabelecimentos escolares brasileiros [Tese de Doutorado]. Belo Horizonte: Centro de Desenvolvimento e Planejamento Regional, Universidade Federal de Minas Gerais; 2004.

22. Krentz HB, Kliewer G, Gill MJ. Changing mortality rates and causes of death for HIV-infected individuals in Southern Albert, Canada from 1984 to 2003. HIV Med 2005; 6:99-106.

23. Yang CY, Su TP, Wong WW, Guo WY, Su YL. Association of AIDS and bipolar mania with rapid progression to dementia and death. J Chin Med Assoc 2005; 68:92-5.

24. Dromer F, Mathoulin-Pelissier S, Fontanet A, Ronin O, Dupont B, Lortholary O, et al. Epidemiology of HIV-associated Cryptococcus in France (19852001): comparison of the pre- and post- HAART eras. AIDS 2004; 18:555-62.

25. Ridolfo AL, Rusconi S, Antinori S, Balotta C, Galli M. Persisting HIV-1 replication triggered by acute hepatitis A virus infection. Antivir Ther 2000; 5 : 15-7.

26. Villarinho L, Bezerra I, Lacerda R, Latorre MDRO, Paiva V, Stall R, et al. Caminhoneiros de rota curta e vulnerabilidade ao HIV, Santos, SP. Rev Saúde Pública 2002; 36 (4 Suppl):61-7.

27. Liberato IRO, Albuquerque MFPM, Campelo ARL, Melo HRL. Characteristics of pulmonary tuberculosis in HIV seropositive and seronegative patients in a northeastern region of Brazil. Rev Soc Bras Med Trop 2004; 37:46-50.

28. Ofotokun I, Pomeroy C. Sex differences in adverse reactions to antiretroviral drugs. Top HIV Med 2003; 11:55-9.

29. Havlir DV, Currier JS. Complications of HIV infection and antiretroviral therapy. Top HIV Med 2003; 11:86-91.

30. Fan H, Conner RF, Villareal LP. The biology of AIDS. Boston: Jones and Barlett Publishers; 1999.

Recebido em 25/Jul/2005

Versão final reapresentada em 19/Jan/2006

Aprovado em 31/Ago/2006 